\title{
Successful Use of Lower-Level Laser Therapy in the Treatment of Medication-Related Osteonecrosis of the Jaw
}

\author{
Gustavo Antonio Correa Momesso' ${ }^{1}$, Fábio Roberto de Souza Batista ${ }^{1}$, Cecília Alves de Sousa ${ }^{1}$, Valthierre \\ Nunes de Lima' ${ }^{1}$ Tárik Ocon Braga Polo', Jaqueline Suemi Hassumi', Idelmo Rangel Garcia Júnior ${ }^{1}$, Leonardo \\ Perez Faverani ${ }^{1}$
}

${ }^{1}$ Department of Surgery and Integrated Clinic, Araçatuba Dental School, Univ. Estadual Paulista (UNESP), Araçatuba, São Paulo, Brazil

\author{
*Correspondence to \\ Gustavo Antonio Correa Momesso, \\ MSc; Department of Surgery and \\ Integrated Clinic, Aracatuba Dental \\ School, Univ. Estadual Paulista \\ (UNESP), Araçatuba, São Paulo, \\ Brasil. \\ Rua José Bonifácio, 1193, \\ Araçatuba, São Paulo, Brasil. \\ Fax: +55 1836363237 \\ Tel: +551836363200 \\ Email: gustavomomesso@gmail.com
}

Published online 27 September 2017

Introduction

Osteonecrosis of the jaws associated with the use of bisphosphonates is one of the most serious complications of long-term therapy with bisphosphonates associated to oral surgical procedures, such as dental implants placement. ${ }^{1}$ Osteonecrosis is defined as bone exposure in the maxillofacial region that does not heal within 8 weeks after its identification. It is associated with the use of an antiresorptive agent (bisphosphonate or denosumab), without history of radiation therapy for the craniofacial region. ${ }^{2}$ The treatment of this condition is extremely difficult. Currently, antibiotic therapy, minimally invasive surgery, and lower-level laser therapy (LLLT) during the early stages have been considered the gold standard for medication-related osteonecrosis of the jaw (MRONJ). ${ }^{3}$

\section{Case Presentation}

A 65-year-old female patient was referred to the oral and maxillofacial surgery team from Araçatuba Dental School 2 months after a dental implant installation, complaining about its mobility. During anamnesis, hypertension and diabetes were reported, both controlled by daily medication, and use of alendronate $(70 \mathrm{mg} / \mathrm{d}$ for 5 years $)$ for prevention of osteoporosis. The clinical examination showed an accentuated mobility of the dental implant in the region of tooth 16 , bone exposure in the periimplant region with vestibular and palatal extensions, purulent secretion and bad odor, and absence of remission of signs and symptoms (Figure 1A). After careful evaluation of the medical history and clinical examination, stage $2 \mathrm{MRONJ}$ was diagnosed, characterized by the presence of exposure and necrotic bone associated with symptomatic infection and purulent discharge. The treatment proposed for this case was the implant removal (Figure 1B), followed by the initiation of 3 sessions per week of LLLT in the area of necrosis over 8 weeks, associated with administration of clindamycin (300 mg every 8 houes) and regular mouthwash with chlorhexidine $0.12 \%$ for the same period. An InGaAlP laser was used (Photon lase, DMC; wavelength $810 \mathrm{~nm}$, power $100 \mathrm{~mW}$, frequency 50/60 $\mathrm{Hz}$, and power density $0.3-0.5 \mathrm{~W} / \mathrm{cm}^{2}$ ). The "alveolitis" program was used, corresponding to $50 \mathrm{~J} / \mathrm{cm}^{2}$ and applied at 3 points of the lesion (distobuccal, mesiobuccal, and palatal), 1-2 $\mathrm{mm}$ from the tissue, 3 times in each spot for 

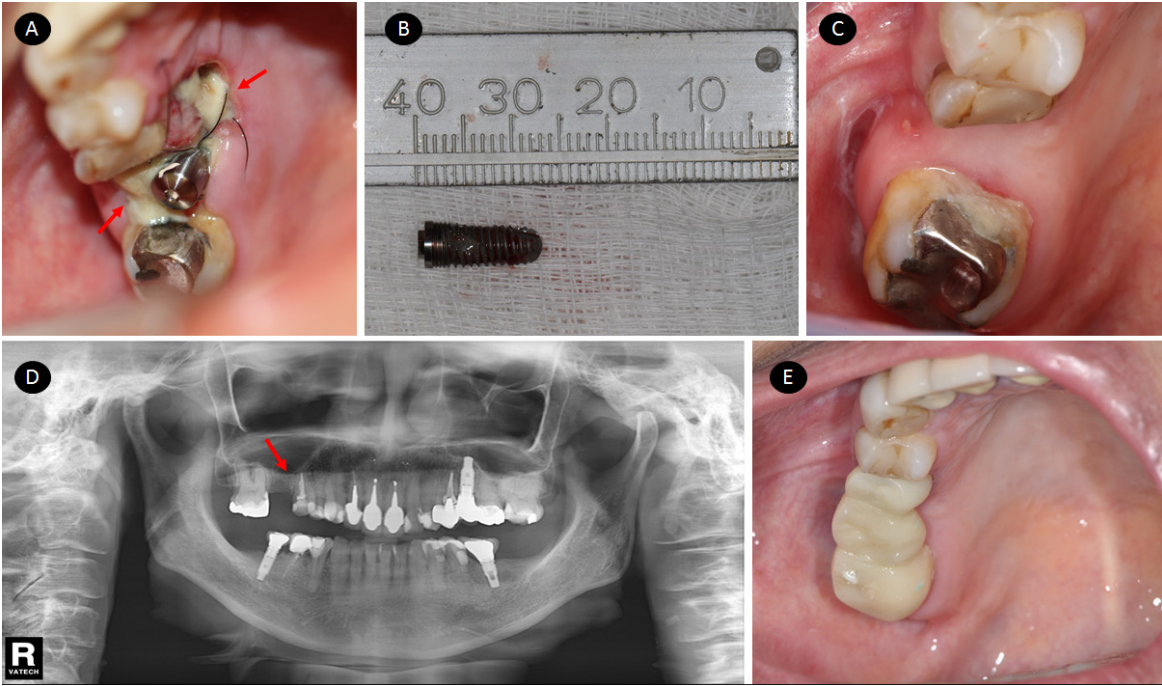

Figure 1. (A) Clinical aspect of MRONJ during examination. It is possible observe necrosis areas affecting the implant region (arrow) extending to the palatal region (arrow). (B) Dental implant removed due necrosis presence and excessive mobility. (C) Clinical aspect on 6 months of follow-up after laser therapy treatment, which shows a complete healing and absence of necrosis areas. (D) Ortopanthomography six months after treatment with laser therapy, showing a great evolution on alveolar healing (arrow). (E) Twelve months after treatment with laser therapy, which shows the final rehabilitation with fixed dental prosthesis, complete healing and no signal of necrosis.

14 seconds each. The "traumatic lesion" program was also used, corresponding to $25 \mathrm{~J} / \mathrm{cm}^{2}$ applied at 3 points of the lesion (distobuccal, mesiobuccal, and palatal), 1-2 mm from the tissue, 3 times in each spot for 7 seconds each. After 8 weeks of laser therapy, significant improvement was observed in the residual repair with cessation of purulent secretion and reduction of the necrosis area. At 6-month follow up after the completion of the laser and antibiotic therapy sessions, complete tissue healing and absence of purulent secretion were noted (Figure 1C). On radiographic examination after this period, it was possible to observe a region of good bone healing in evolution (Figure 1D). Twelve months after treatment was started, the patient was rehabilitated with a fixed dental prosthesis and presented great healing with no complaints (Figure 1E).

\section{Discussion}

Dentoalveolar surgery for patients using oral bisphosphonates for more than 4 years is considered a risk factor for MRONJ. Thus, the suspension of the drug 2 months before the surgical procedure is proposed. ${ }^{4}$ However, in this case the patient did not report the use of sodium alendronate at the implant placement time. The literature shows evidence of high rates of implant loss and a high incidence of osteonecrosis in patients submitted to implant surgery associated with the use of bisphosphonates, and the risk of developing osteonecrosis as well as implant failure exists; however, it is greater in patients receiving intravenous bisphosphonates such as zoledronic acid. ${ }^{5}$

Several treatments for MRONJ have been proposed, such as surgical, conservative, and combined surgicalconservative therapies. The treatment indication varies according to the stage of the lesion. The most advanced stages indicate a need for the most aggressive approach. ${ }^{6}$ However, no one has demonstrated total efficacy. ${ }^{2}$ Laser therapy performs very well for patients with at least stage 2 MRONJ. ${ }^{7}$ The patient in this study presented stage 2 MRONJ, thus we opted to use LLLT, which has been shown to be a promising adjuvant treatment for this condition due its ability to modulate the cellular metabolism, improve the wound healing, and relieve pain. The biostimulating effect of laser irradiation expands the organic bone matrix and increases the mitotic index of osteoblasts, stimulating its proliferation and differentiation and increasing the number of differentiated osteoblastic cells and their activity. In addition, LLLT is a noninvasive method with antibacterial and biostimulating effects on soft and hard tissues and presents proangiogenic factors. ${ }^{3}$

This study showed a successful treatment of MRONJ using a protocol composed of antibiotic therapy with clindamycin, minimally invasive surgery, and LLLT. Thus, we conclude that it is extremely important to obtain a complete medical history of a patient, and if bisphosphonate therapy is confirmed, then parameters such as duration of treatment and route of administration should be considered for planning a surgical procedure as guided by the recommendations of the American Association of Oral and Maxillofacial Surgeons (AAOMS).

\section{Ethical Considerations}

Written informed consent was obtained from the patient or for publishing this case report.

\section{Conflict of Interests}

The authors have no conflict of interest to declare. 


\section{References}

1. Svejda B, Muschitz C, Gruber R, et al. Position paper on medication-related osteonecrosis of the jaw (MRONJ) (German). Wien Med Wochenschr. 2016;166(1-2):68-74. doi:10.1007/s10354-016-0437-2.

2. Ruggiero SL, Dodson TB, Fantasia J, et al. American Association of Oral and Maxillofacial Surgeons position paper on medication-related osteonecrosis of the jaw-2014 update. J Oral Maxillofac Surg. 2014;72(10):19381956. doi:10.1016/j.joms.2014.04.031

3. Weber JB, Camilotti RS, Ponte ME. Efficacy of laser therapy in the management of bisphosphonate-related osteonecrosis of the jaw (BRONJ): a systematic review. Lasers Med Sci. 2016;31(6):1261-1272. doi:10.1007/s10103016-1929-4.

4. Khan A, Morrison A, Cheung A, Hashem W, Compston J.
Osteonecrosis of the jaw (ONJ): diagnosis and management in 2015. Osteoporos Int. 2016;27(3):853-859. doi:10.1007/ s00198-015-3335-3.

5. de-Freitas NR, Lima LB, de-Moura MB, Veloso-Guedes CC, Simamoto-Júnior PC, de-Magalhães D. Bisphosphonate treatment and dental implants: A systematic review. Med Oral Patol Oral Cir Bucal. 2016;21(5):e644-e651.

6. Ruggiero SL. An office-based approach to the diagnosis and management of osteonecrosis. Atlas Oral Maxillofac Surg Clin North Am. 2013;21(2):167-173. doi:10.1016/j. cxom.2013.05.004.

7. Vescovi P, Manfredi M, Merigo E, et al. Early surgical laser-assisted management of bisphosphonate-related osteonecrosis of the jaws (BRONJ): a retrospective analysis of 101 treated sites with long-term follow-up. Photomed Laser Surg. 2012;30(1):5-13. doi:10.1089/pho.2010.2955. 